\title{
Fractional Calculus, Fractional Differential Equations and Applications
}

\author{
Mariam Almahdi Mohammed Mu'lla ${ }^{1,2}$ \\ ${ }^{1}$ Department of Mathematics, University of Hafr Al-Batin (UoHB), Hafr Albatin, KSA \\ ${ }^{2}$ University of Kordofan, El-Obeid, North Kordofan, Sudan \\ Email: marimdx2014@gmail.com
}

How to cite this paper: Mu'lla, M.A.M. (2020) Fractional Calculus, Fractional Differential Equations and Applications. Open Access Library Journal, 7: e6244. https://doi.org/10.4236/oalib.1106244

Received: March 18, 2020

Accepted: June 20, 2020

Published: June 23, 2020

Copyright $\odot 2020$ by author(s) and Open Access Library Inc.

This work is licensed under the Creative Commons Attribution International License (CC BY 4.0).

http://creativecommons.org/licenses/by/4.0/

\begin{abstract}
In this paper, we describe two approaches to the definition of fractional derivatives. We investigate the accuracy of the analysis method for solving the fractional order problem. We also give some improvements for the proof of the existence and uniqueness of the solution in fractional differential equations. Treatment of a fractional derivative operator has been made associated with the extended Appell hypergeometric functions of two or three variables and Lauricella hypergeometric function of three variables.
\end{abstract}

\section{Subject Areas \\ Mathematical Analysis, Mathematical Algebra and Foundation of Mathematics}

\section{Keywords}

Fractional Derivative, Fractional Differentiation, Factorial for the Integer Numbers, Riemann-Liouville Fractional Derivative

\section{Introduction}

For compressible flows, in particular, there are additional degrees of fractional differential equations and treatment of a fractional derivative operator [1]. The latter can have very different characteristic amplitudes and scales in comparison with the vorticity field. Fractional-order systems are useful in studying the anomalous behavior of dynamical systems in physics, and electrochemistry. A particular difficulty of modeling such flows at high Reynolds (Re) numbers is the diversity of space and time scales that emerge as the flow develops. A particular difficulty of modeling such flows at high Reynolds $(\mathrm{Re})$ numbers is the diversity of space and time scales that emerge as the flow develops fractional order models 
of neurons, the electric conductance of biological systems, fitting experimental data, and medical analysis of special functions (see [2] [3]). In case of high re-number flows, the disparity of the scales happens. We launch a new Riemann-Liouville fractional derivative operator associated with hypergeometric type function [4]. Further, we investigate some properties of the new fractional derivative operator. For engineering applications, examples of unsteady vertical flows include the interaction of wakes and shocks with the boundary layer in a transonic turbine and vorticity dissipation shed due to the temporal variations in blade circulation that can have a profound loss influence and affect the overall performance of a turbomachine (e.g., Fritsch and Giles, 1992; Michelassi et al., 2003). Another example is dynamics and acoustics of high-speed jet flows that are affected by the jet inflow conditions such as the state of the boundary layer at the nozzle exit (e.g., Bogey and Bailly, 2010). The computational aspects involved in the modelling of such complex flows, typically, include the issues of high-resolution numerical schemes, boundary conditions, non-uniform grids and the choice of sub grid scale parameterization. Fractional calculus is allowing integrals and derivatives of any positive order (the term fractional is kept only for historical reasons) [5] [6] [7]. It can be considered a branch of mathematical physics that deals with integral-differential equations, where integrals are of convolution type and exhibit mainly singular kernels of power law or logarithm type. The purpose of this Special Issue is to establish a collection of articles that reflect the latest mathematical and conceptual developments in the field of fractional calculus and explore the scope for applications in applied sciences [8] [9].

\section{Differential Equations of Fractional Order}

The fractional ordinary differential equations have the following general form

$$
F\left(x, y(x), D^{\alpha_{1}} y(x), D^{\alpha_{2}} y(x), \cdots, D^{\alpha_{n}} y(x)\right)=g(x)
$$

where $F\left(x, y_{1}, y_{2}, \cdots, y_{n}\right)$ and $g(x)$ are given function and $D^{\alpha_{n}}$ are the operators of fractional differentiation with real $\alpha_{n}>0$ or complex $\alpha_{n}$, $\operatorname{Re}_{n}>0, n=1,2,3, \cdots, k$. for the nonlinear differential

$$
D^{\alpha} y(x)=f(x, y(x))
$$

With real $\alpha>0$ or complex $\alpha(\operatorname{Re}(\alpha)>0)$, and the linear differential equations

$$
\sum_{n=1}^{k} c_{n}(x) D^{\alpha_{n}} y(x)+c_{0}(x) y(x)=f(x)
$$

The fractional differentiation operators (1) and (3) can have different forms see [2], but the equations containing the Riemann Liouville fractional derivative are studied. For complex $\alpha \in \mathbb{C}, \operatorname{Re}(\alpha)>0$, such a fractional derivative is defined by

$$
\left(D_{\alpha+}^{\alpha} y\right)(x)=\left(\frac{\mathrm{d}}{\mathrm{d} x}\right)^{n}\left(I_{\alpha+}^{n-\alpha} y\right)(x), n=[\operatorname{Re}(\alpha)]+1,
$$

where $\left(I_{\alpha+}^{n-\alpha} y\right)(x)$ is the fractional integral of order $\alpha$. 


$$
\left(I_{\alpha+}^{n-\alpha} y\right)(x)=\frac{1}{\Gamma(x)} \int_{\alpha}^{x} \frac{y(t) \mathrm{d} t}{(x-t)^{1-\alpha}}(\alpha \in \mathbb{C}, \operatorname{Re}(\alpha)>0)
$$

$\Gamma(x)$ is the Gamma function [4] [10]. It should be noted that the Riemann Liouville approach (5) to the definition of fractional integration is a generalization of the integration operator $\int_{\alpha+}^{x}$ applied $n$ times:

$$
\int_{\alpha}^{x} \mathrm{~d} t \int_{\alpha}^{t} \mathrm{~d} t_{1} \cdots \int_{\alpha}^{t_{n-2}} y\left(t_{n-1}\right) \mathrm{d} t_{n-1}=\frac{1}{(n-1) !} \int_{\alpha}^{x}(x-t)^{n-1} y(t) \mathrm{d} t
$$

(If we use the formula $(n-1) !=\Gamma(n))$ and replaced $n$ by $\alpha \in \mathbb{C} \quad(\operatorname{Re}(\alpha)>0)$, then (6) yields (5) and the fractional differentiation operator $D_{\alpha+}^{\alpha}$ is inverse to the fractional integration one from the left:

$$
\left(D_{\alpha+}^{\alpha} I_{\alpha+}^{\alpha}\right)(x)=y(x)(\alpha \in \mathbb{C}, \operatorname{Re}(\alpha)>0)
$$

For suitable function $y(x)$ from (5). For real $\alpha>0$, Equation (4) takes the form

$$
\left(D_{\alpha+}^{\alpha} I_{\alpha+}^{\alpha}\right)(x)=\left(\frac{\mathrm{d}}{\mathrm{d} x}\right)^{n} \frac{1}{\Gamma(n-\alpha)} \int_{\alpha}^{x} \frac{y(t) \mathrm{d} t}{(x-t)^{\alpha-n+1}}, \quad n=[\alpha]+1,
$$

where $[\alpha]$ means the integral part of $\alpha$ in particular if $0<\alpha<1$,

$$
\left(D_{\alpha+}^{\alpha} y\right)(x)=\frac{\mathrm{d}}{\mathrm{d} x} \frac{1}{\Gamma(1-\alpha)} \int_{\alpha}^{x} \frac{y(t) \mathrm{d} t}{(x-t)^{\alpha}},
$$

And if $\alpha=n \in \mathbb{N}=\{1,2, \cdots\}$, then $\left(D_{\alpha+}^{\alpha} y\right)(x) \equiv\left(D^{n} y\right)(x),\left(D=\frac{\mathrm{d}}{\mathrm{d} x}\right)$ is the usual derivative of order $n[2]$.

\section{Fractional Calculus, Fractional Differential Equations and Applications}

In mathematics, many complex concepts developed from simple concepts. For example, we can refer to the extension of natural number to the real one in some mathematical formulae. Let's give an example to clarify: the factorial of a non-negative integer $n$ [11] [12] [13], denoted by $n$ !, is the product of all positive integers less than or equal to $n$. On the other hand, there is a concept named Gama function and defined as follows

$$
\Gamma(x)=\int_{0}^{\infty} t^{x-1} \mathrm{e}^{-t} \mathrm{~d} t
$$

One property of the function for $n \in \mathbb{R}^{+}$is

$$
\Gamma(n+1)=n \Gamma(n)
$$

Hence, this function is equal to factorial for the integer numbers. As a result, the gamma function could be considered as an extension of factorial function to real numbers. For instance, according to the above formalism, a factorial of $1 / 2$ can be obtained as follows:

$$
\left(\frac{1}{2}\right) !=\Gamma\left(\frac{3}{2}\right)=\left(\frac{3}{2}\right) \Gamma\left(\frac{1}{2}\right)=\frac{3 \sqrt{\pi}}{2}
$$


According to Wikipedia, the gamma function can be seen as the solution to find a smooth curve that connects the points $(x, y)$ given by $y=(x-1) !$ at the positive integer values for $x$.

Lemma 3.1. Let $1<\alpha \leq 2$. The following relations hold:

1) if $y \in L^{\infty}(J)$, then $\mathbb{D}_{c}^{\alpha} I^{\alpha} y(t)=y(t)$,

2) if $y \in A C^{1}(J)$, then

$$
I^{\alpha c} \mathbb{D}^{\alpha} y(t)=y(t)-y(0)-y^{\prime}(0) t .
$$

then $y \in A C^{1}(J)$ is a solution of the boundary value problem

$$
\mathbb{D}_{c}^{\alpha} y(t)+\mu(t)=0, t \in J, y(0)=y_{0}, y(1)=y_{1},
$$

if and only if $y$ satisfies:

$$
y(t)=\int_{0}^{1} G(t, z) \mu(z) \mathrm{d} z+\left(y_{1}-y_{0}\right) t+y_{0}, t \in J,
$$

where the Green's function associated with (1) is defined by

$$
G(t, z)=\frac{1}{\Gamma(\alpha)} \begin{cases}t(1-z)^{\alpha-1}-(t-z)^{\alpha-1}, & 0 \leq z \leq t \leq 1 \\ t(1-z)^{\alpha}, & 0 \leq z \leq t \leq 1\end{cases}
$$

Proof By Lemma 2.1 (2), we deduce from equation (13) that

$$
0=I^{\alpha c} \mathbb{D}^{\alpha} y(t)+I^{\alpha} \mu(t)=y(t)-y^{\prime}(0)+\int_{0}^{t} \frac{(t-z)^{\alpha-1}}{\Gamma(\alpha)} \mu(z) d z,
$$

and the boundary conditions give:

$$
y(0)=y_{0}, \quad y^{\prime}(0)=y_{1}-y_{0}+\int_{0}^{t} \frac{(t-z)^{\alpha-1}}{\Gamma(\alpha)} \mu(z) \mathrm{d} z,
$$

Thus, we obtain that:

$$
\begin{aligned}
y(t) & =-\int_{0}^{t} \frac{(t-z)^{\alpha-1}}{\Gamma(\alpha)} \mu(z) d z+t \int_{0}^{t} \frac{(t-z)^{\alpha-1}}{\Gamma(\alpha)} \mu(z) \mathrm{d} z+\left(y_{1}-y_{0}\right) t+y_{0} \\
& =\int_{0}^{t} G(t, z) \mu(z) \mathrm{d} z+\left(y_{1}-y_{0}\right) t+y_{0}, \quad t \in J .
\end{aligned}
$$

Inversely, if $y \in A C^{1}(J)$ satisfies (2), then we have:

$$
y(t)=-I^{\alpha} \mu(t)+\left(\int_{0}^{t} \frac{(t-z)^{\alpha-1}}{\Gamma(\alpha)} \mu(z) \mathrm{d} z+y_{1}-y_{0}\right) t+y_{0} .
$$

(which shows that $\mathbb{D}_{c}^{\alpha} t=\mathbb{D}_{c}^{\alpha} 1=0$ ) we have:

$$
\mathbb{D}_{c}^{\alpha} y(t)=-\mu(t), t \in J
$$

that is, $y$ is a proof of (1) [14].

Definition 3.2 If $\alpha \in \mathbb{R}$, then the fractional derivative $\alpha D$ exists almost everywhere on $\mathbb{D}$. If $\alpha>0$ is not an integer, then it is expressed as follows:

$$
\left(\mathbb{D}_{c}^{\alpha} \mu\right)(t)=\frac{1}{\Gamma(n-\alpha)} \int_{0}^{t}(t-z)^{n-\alpha-1} \mu^{(n)}(z) \mathrm{d} z
$$


where $n=[\alpha]+1$ and $[\alpha]$ stands for the largest integer not greater than $\alpha[9]$.

Let's use this approach to extend the concept of derivative to non-integer order; consider $n$th derivation of power function $g(x)$.

$$
\begin{gathered}
g(x)=x^{k}, \quad x \geq 0 \\
\frac{\mathrm{d}^{n}}{\mathrm{~d} x^{n}} g(x)=\frac{k !}{(k-n) !} x^{k-n}=\frac{\Gamma(1+k)}{\Gamma(1+k-n)} x^{k-n}
\end{gathered}
$$

where $k$ and $n$ are real integer number respectively, and $k \geq n$. To generalize the above equation, it could be possible to extend the integer number $n$ to a real value named $\alpha$ :

$$
\frac{\mathrm{d}^{\alpha}}{\mathrm{d} x^{\alpha}} g(x)=\frac{\Gamma(1+k)}{\Gamma(1+k-\alpha)} x^{k-\alpha}
$$

Then for fractional derivative of an arbitrary function, expand the function in a power series of $x$ first, and then by using equation (18) derivate the expansion. For example, for derivative $f(x)=\mathrm{e}^{k x}$ to $\alpha$ order, we rewrite $f(x)$ function as follows:

$$
f(x)=1+x+\frac{x^{2}}{2 !}+\frac{x^{3}}{3 !}+\cdots
$$

Hence

$$
\begin{aligned}
\frac{\mathrm{d}^{\alpha}}{\mathrm{d} x^{\alpha}} f(x) & =\frac{1}{\Gamma(1-\alpha)} x^{-\alpha}+\frac{1}{\Gamma(2-\alpha)} x^{1-\alpha}+\frac{1}{\Gamma(3-\alpha)} x^{3-\alpha}+\cdots \\
& =\operatorname{sign}(x)(\operatorname{sign}(x) k)^{\alpha} \mathrm{e}^{k x}\left(1-\frac{\Gamma(-\alpha, k x)}{\Gamma(2 \alpha)}\right)
\end{aligned}
$$

That $\Gamma(-\alpha, k x)$ is incomplete gamma function [3] [9] [15] [16] [17] [18].

This is an arbitrary way to define fractional derivative and not the only way, for example, it is possible to use an exponential function $f(x)=\mathrm{e}^{k x}$ instead of a power function, we can define:

$$
D^{\alpha} f(x)=k^{\alpha} \mathrm{e}^{k x}
$$

The fractional derivative of the exponential function obtained by Liouville in 1832 [15], and the fractional derivative of power function got by Riemann in $1847[16]$.

\section{Riemann-Liouville Fractional Derivative}

In this section, we shall exploit the concept of Riemann-Liouville type fractional derivative operator [17] [19]. For this purpose, we first consider the Riemann Liouville fractional derivative of $f(z)$ of order $v$ as follows:

$$
\mathbb{D}_{z}^{v}\{f(z)\}=\int_{0}^{z}(z-t)^{-v-1} f(t) \mathrm{d} t, \mathcal{R}(v)<0
$$

where the integration path is a line from 0 to $z$ in the complex $t$-plane [10] [20] [21].

The Riemann-Liouville fractional derivative of $f(z)$ of order $v$ is defined as: 


$$
\begin{aligned}
\mathbb{D}_{z}^{v}\{f(z)\} & =\frac{\mathrm{d}^{m}}{\mathrm{~d} z^{m}} \mathbb{D}_{z}^{v-m}\{f(z)\} \\
& =\frac{\mathrm{d}^{m}}{\mathrm{~d} z^{m}}\left\{\frac{1}{\Gamma(-v+m)} \int_{0}^{z}(z-t)^{-v+m-1} f(t) \mathrm{d} t\right\}, \mathcal{R}(v)<0
\end{aligned}
$$

When $\mathcal{R}(v)$, let $m \in \mathbb{N}$ be the smallest integer greater than $\mathcal{R}(v)$ and so $m-1 \leq \mathcal{R}(v)<m$, then a new Riemann-Liouville fractional derivative of $f(z)$ of order $v$ can be defined as follows:

$$
\begin{aligned}
& \mathbb{D}_{z}^{v,[p]_{q}}\{f(z)\}=\frac{\mathrm{d}^{m}}{\mathrm{~d} z^{m}} \mathbb{D}_{z}^{v-m,[p]_{q}}\{f(z)\} \\
& =\frac{\mathrm{d}^{m}}{\mathrm{~d} z^{m}}\left\{\frac{\sqrt{\frac{2 p}{\pi}}}{\Gamma(-v+m)} \int_{0}^{z} f(t)(z-t)^{-v+m-\frac{3}{2}} K_{q+\frac{1}{2}}\left(\frac{p z^{2}}{t(z-t)}\right) \mathrm{d} t\right\}, \\
& (\Re(p)>0, \Re(q)>0)
\end{aligned}
$$

Remark On setting $p=0, q=0$ in (18) and (19) we are left with the classical Riemann-Liouville fractional derivative. In the case $q=0$ in Equations (24) and (25) reduces to the well-known fractional derivative operator given in [16] [22] [23].

\section{Fractional Derivative of Some Functions}

\section{Theorem 5.1}

Let $m-1 \leq \mathfrak{R}(v)<m<R(\lambda)$ for some $m \in \mathbb{N}$. Suppose that a function $f(z)$ is analytic at the origin with its Maclaurin expansion given by $f(z)=\sum_{n=0}^{\infty} a_{n} z^{n},(|z|<\xi)$ for some $\xi \in \mathbb{R}^{+}$. Then we have

$$
\mathbb{D}_{z}^{v,[p]_{q}}\left\{z^{\lambda-\frac{3}{2}} f(z)\right\}=\frac{z^{\lambda-v-2}}{\Gamma(-v)} \sum_{n=0}^{\infty} a_{n} B_{p, q}(\lambda+n,-v) z^{n} .
$$

Proof Now applying (24) in the definition (25) to the function $z^{\lambda-\frac{3}{2}} f(z)$, and changing the order of integration and summation, we obtain

$$
\begin{aligned}
& \mathbb{D}_{z}^{v,[p]_{q}}\left\{z^{\lambda-\frac{3}{2}} f(z)\right\} \\
& =\frac{\sqrt{\frac{2 p}{\pi}}}{\Gamma(-v)} \sum_{n=0}^{\infty} a_{n} \int_{0}^{z} t^{\lambda+n-\frac{3}{2}}(z-t)^{-v-\frac{3}{2}} K_{q+\frac{1}{2}}\left(\frac{p z^{2}}{t(z-t)}\right) \mathrm{d} t
\end{aligned}
$$

Putting $t=\xi_{Z}$ in (27), we obtain

$$
\begin{aligned}
& \mathbb{D}_{z}^{v,[p]_{q}}\left\{z^{\lambda-\frac{3}{2}} f(z)\right\} \\
& =\frac{z^{\lambda-v-2} \sqrt{\frac{2 p}{\pi}}}{\Gamma(-v)} \sum_{n=0}^{\infty} a_{n} z^{n} \int_{0}^{1} \xi^{\lambda+n-\frac{3}{2}}(1-\xi)^{-v-\frac{3}{2}} K_{q+\frac{1}{2}}\left(\frac{p}{\xi(z-\xi)}\right) \mathrm{d} \xi .
\end{aligned}
$$

The applying definition of extended beta function, and after some simplifica- 
tion, we get the desired result as follows:

$$
\begin{aligned}
& \mathbb{D}_{z}^{v,[p]_{q}}\left\{z^{\lambda-\frac{3}{2}} \log z f(z)\right\} \\
& =\sum_{n=0}^{\infty} z^{\lambda+n-v-2}\left\{a_{n} \log (z) B_{p, q}(\lambda+n, v)+b_{n} B_{p, q}(\lambda+n,-v+1)\right\},
\end{aligned}
$$

Example 5.2 Let $n-1 \leq(v)<n<(\lambda)$ for some $m \in \mathbb{N}$. Then we have

$$
\mathbb{D}_{z}^{v,[p]_{q}}\left\{z^{\lambda}\right\}=\frac{B_{p, q}\left(\lambda+\frac{3}{2}-v\right)}{\Gamma(-v)} z^{\lambda-v-2}
$$

Solution We have applied the definition of the fractional derivative, we obtain

$$
\mathbb{D}_{z}^{v,[p]_{q}}\left\{z^{\lambda}\right\}=\frac{\sqrt{\frac{2 p}{\pi}}}{\Gamma(-v)} \int_{0}^{z} f(t)(z-t)^{-v+m-\frac{3}{2}} K_{q+\frac{1}{2}}\left(\frac{p z^{2}}{t(z-t)}\right) \mathrm{d} t
$$

Putting $t=\mu z$ in (24), we obtain

$$
\mathbb{D}_{z}^{v,[p]_{q}}\left\{z^{\lambda}\right\}=\frac{z^{\lambda-v-\frac{1}{2}} \sqrt{\frac{2 p}{\pi}}}{\Gamma(-v)} \int_{0}^{1} \mu^{\lambda}(z-\mu)^{-v+-\frac{3}{2}} K_{q+\frac{1}{2}}\left(\frac{p}{\mu(z-\mu)}\right) \mathrm{d} \mu
$$

We apply the definition of the extended beta function, we obtain the solution [21] [24].

Example 5.3 Let $n-1 \leq(v)<n<(\lambda)$ for some $m \in \mathbb{N}$. Then we have

$$
\mathbb{D}_{z}^{\lambda-v,[p]_{q}}\left\{z^{\lambda-\frac{3}{2}}(1-z)^{-\alpha}\right\}=\frac{\Gamma(\lambda)}{\Gamma(v)} G_{p, q}(\alpha, \lambda, v, z) z^{v-2} .
$$

Solution Applying the definition of the new fractional derivative operator, we obtain

$$
\begin{aligned}
& \mathbb{D}_{z}^{\lambda-v,[p]_{q}}\left\{z^{\lambda-\frac{3}{2}}(1-z)^{-\alpha}\right\} \\
& =\frac{\sqrt{\frac{2 p}{\pi}}}{\Gamma(-v)} \int_{0}^{1} z^{\lambda-\frac{3}{2}}(1-t)^{-\alpha}(z-t)^{v-\lambda-\frac{3}{2}} K_{q+\frac{1}{2}}\left(\frac{p z^{2}}{t(z-t)}\right) \mathrm{d} t .
\end{aligned}
$$

Putting $t=\mu z$ in (27), we obtain

$$
\begin{aligned}
& \mathbb{D}_{z}^{\lambda-v,[p]_{q}}\left\{z^{\lambda-\frac{3}{2}}(1-z)^{-\alpha}\right\} \\
& =\frac{z^{v-2} \sqrt{\frac{2 p}{\pi}}}{\Gamma(v-\lambda)} \int_{0}^{1} \mu^{\lambda-\frac{3}{2}}(1-\mu)^{-\alpha}(z-z \mu)^{-\alpha} K_{q+\frac{1}{2}}\left(\frac{p}{\mu(z-\mu)}\right) \mathrm{d} \mu .
\end{aligned}
$$

We apply the definition of the extended hypergeometric function, we get the solution [2] [24].

\section{Conclusions}

In this paper, we have special issue to establish a collection of functions in the 
field of fractional calculus and explore the scope for applications in applied sciences. We have also defined fractional calculus, fractional differential, and an interesting Riemann-Liouville fractional derivative operator. In addition to that, we have important properties of the new fractional derivative operator. And we apply the definition of the new fractional derivative operator. We define the extended beta function that we obtained.

As an application of our new operator, we have established some interesting generating functions for the extended hypergeometric function $F_{p, q}$, using the new operator.

\section{Acknowledgements}

I would like to thank my supervisor, Dr. Muhsin Hassan Abdallah who was a great help to me and also I thanks my husband Bashir Alfadol Albdawi without whose help, I could not have written this paper.

\section{Conflicts of Interest}

The author declares no conflicts of interest regarding the publication of this paper.

\section{References}

[1] Caponetto, R. (2010) Fractional Order Systems (Modelling and Control Applications). World Scientific, Singapore. https://doi.org/10.1142/7709

[2] Samko, S.G., Kilbas, A.A. and Marichev, O.I. (1993) Fractional Integrals and Derivatives: Theory and Applications. Gordon and Breach, Yverdon.

[3] Olver, F.W.J., Lozier, D.W., Boisvert, R.F. and Clark, C.W. (2010) NIST Handbook of Mathematical Functions [with 1 CD-ROM (Windows, Macintosh and UNIX)]. Cambridge University Press, Cambridge.

[4] Erdelyi, A., Magnus, W., Oberhettinger, F. and Tricomi, F.G. (1981) Higher Transcendental Functions. Vol. 1, McGraw-Hill Book Corp., New York.

[5] Diethelm, K. (2010) The Analysis of Fractional Differential Equations, an Application Oriented, Exposition Using Differential Operators of Caputo Type. Lecture Notes in Mathematics, Springer, Berlin. https://doi.org/10.1007/978-3-642-14574-2 8

[6] Hilfer, R. (2003) Applications of Fractional Calculus in Physics. World Scientific, Singapore.

[7] Kilbas, A.A., Srivastava, H.M. and Trujillo, J.J. (2006) Theory and Applications of Fractional Differential Equations. North-Holland, Amsterdam.

[8] Agarwal, P., Nieto, J.J. and Luo, M.J. (2017) Extended Riemann-Liouville Type Fractional Derivative Operator with Applications. Applied Mathematics \& Information Sciences, 15, 12-29. https://doi.org/10.1515/math-2017-0137

[9] Rainville, E.D. (1971) Special Functions. Macmillan Co., New York.

[10] Chatterjee, A. (2005) Statistical Origins of Fractional Derivatives in Viscoelasticity. Journal of Sound and Vibration, 284, 1239-1245. https://doi.org/10.1016/j.jsv.2004.09.019

[11] Diethelm, K. and Ford, N.J. (2004) Multi-Order Fractional Differential Equations 
and Their Numerical Solution. Applied Mathematics and Computation, 154, 621-640. https://doi.org/10.1016/S0096-3003(03)00739-2

[12] Kochubei, A.N. (2009) Fractional Differential Equations: $\alpha$-Entire Solutions, Regular and Irregular Singularities. Fractional Calculus and Applied Analysis, 12, 135-158.

[13] Lubich, C. (1986) Discretized Fractional Calculus. SIAM Journal on Mathematical Analysis, 17, 704-719. https://doi.org/10.1137/0517050

[14] Kilbas, A.A., Srivastava, H.M. and Trujillo, J.J. (2006) Theory and Applications of Fractional Differential Equations. Elsevier, Amsterdam.

[15] Diethelm, K. (2007) Smoothness Properties of Solutions of Caputo-Type Fractional Differential Equations. Fractional Calculus and Applied Analysis, 10, 151-160.

[16] Herrmann, R. (2011) Fractional Calculus: An Introduction for Physicists. World Scientific, Singapore. https://doi.org/10.1142/8072

[17] Mainardi, F. (2010) Fractional Calculus and Waves in Linear Viscoelasticity: An Introduction to Mathematical Models. World Scientific, Singapore. https://doi.org/10.1142/p614

[18] Diethelm, K. (2008) Multi-Term Fractional Differential Equations, Multi-Order Fractional Differential Systems and Their Numerical Solution. Journal Européen des Systèmes Automatisés, 42, 665-676. https://doi.org/10.3166/jesa.42.665-676

[19] Miller, K.S. and Ross, B. (1993) An Introduction to Fractional Calculus and Fractional Differential Equations. Wiley, New York.

[20] Baleanu, D., Guvenc, Z.B. and Machado, J.A.T. (2010) New Trends in Nanotechnology and Fractional Calculus Applications. Springer, Berlin.

https://doi.org/10.1007/978-90-481-3293-5

[21] Luchko, Y. and Gorenflo, R. (1999) An Operational Method for Solving Fractional Differential Equations with the Caputo Derivatives. Acta Mathematica Vietnamica, 24, 207-233.

[22] Baleanu, D., Agarwal, P., Parmar, R.K., Alqurashi, M.M. and Salahshour, S. (2015) Extended Riemann-Liouville Fractional Derivative Operator and Its Applications. Journal of Nonlinear Sciences and Applications, 10, 2914-2924. https://doi.org/10.22436/jnsa.010.06.06

[23] Özarslan, M.A. and Özergin, E. (2010) Some Generating Relations for Extended Hypergeometric Functions via Generalized Fractional Derivative Operator. Mathematical and Computer Modelling, 52, 1825-1833. https://doi.org/10.1016/j.mcm.2010.07.011

[24] Magin, R.L. (2011) Fractional Calculus Models of Complex Dynamics in Biological Tissues. Computers \& Mathematics with Applications, 59, 1586-1593. https://doi.org/10.1016/j.camwa.2009.08.039 